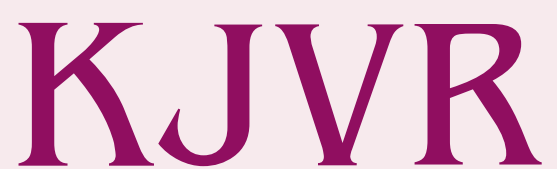

Korean Journal of Veterinary Research

\section{Case Report}

pISSN 2466-1384 · elSSN 2466-1392

Korean J Vet Res 2021;61(1):e3

https://doi.org/10.14405/kjvr.2021.61.e3

*Corresponding author:

Kija Lee

Department of Veterinary Medical

Imaging, College of Veterinary Medicine,

Kyungpook National University, 80

Daehak-ro, Buk-gu, Daegu 41566, Korea

Tel: +82-53-950-5961

Fax: +82-53-950-5955

E-mail: leekj@knu.ac.kr

ORCID:

https://orcid.org/0000-0002-4649-809X

Conflict of interest:

The authors declare no conflict of interest.

Received: October 27, 2020

Revised: December 30, 2020

Accepted: January 15, 2021

\title{
Hypertrophic pyloric gastropathy with Helicobacter spp. in a dog
}

Hyunyoung Jang ${ }^{1}$, Seoung-Woo Lee ${ }^{2}$, Min Jang ${ }^{3}$, Sungho Yun ${ }^{3}$, Young-Sam Kwon ${ }^{3}$, Jin-Kyu Park², Kija Lee ${ }^{1, *}$

1 Department of Veterinary Medical Imaging, College of Veterinary Medicine, Kyungpook National University, Daegu 41566, Korea

${ }^{2}$ Department of Veterinary Pathology, College of Veterinary Medicine, Kyungpook National University, Daegu 41566, Korea

${ }^{3}$ Department of Veterinary Surgery, College of Veterinary Medicine, Kyungpook National University, Daegu 41566, Korea

A 12-year-old, castrated male, mixed dog presented with a history of gradual abdominal distention for a year and anorexia recently, with abdominal radiographs showing a gastric pylorus distention. A solitary, pedunculated, heterogeneous mass arising from the mucosal layer in the pylorus with intact wall layers was identified during ultrasound and computed tomography. The gastric muscular layer was evenly thick. After surgical excision of the mass, histological examination confirmed hypertrophic pyloric gastropathy with polypoid growth and Helicobacter spp. infiltrating the gastric mucosal epithelium. This is the first reported diagnostic imaging case of hypertrophic pyloric gastropathy with Helicobacter spp. in a dog.

Keywords: dogs; hypertrophic pyloric gastropathy; Helicobacter; ultrasonography; diagnostic imaging

Chronic hypertrophic pyloric gastropathy is common in small, purebred, middle- to old-aged dogs [1,2]. Hypertrophic gastropathy is described as a condition of unknown origin but associated with Helicobacter pylori in $90 \%$ of patients in human medicine [3]. The breeds included Maltese, Shih-Tzu, Yorkshire Terrier, and Chihuahua with mean age at presentation of 8.8 years, ranging from 3 to 15 years [1,2]. Approximately twice as many male as female dogs are affected [1], presenting with clinical signs such as chronic vomiting, weight loss, polydipsia, depression, lethargy, anorexia, and abdominal pain [2]. Definitive diagnosis of chronic hypertrophic pyloric gastropathy requires a full-layer biopsy of the stomach, and diagnostic imaging can aid the diagnosis of chronic hypertrophic pyloric gastropathy [1]. But diagnostic imaging reports of hypertrophic pyloric gastropathy are rare, and computed tomography (CT) reports only one case. This report describes a diagnostic imaging and treatment of hypertrophic pyloric gastropathy with Helicobacter spp. in a dog.

A 12-year-old, castrated male, mixed dog weighing $4.85 \mathrm{~kg}$ was referred with a history of gradual abdominal distention for a year and anorexia recently. During physical examination, no significant findings were found except abdominal distention with serum chemistry showing hypochloremia $(99 \mathrm{mmol} / \mathrm{L}$, reference range 102 to $117 \mathrm{mmol} / \mathrm{L}$ ).

Abdominal radiography showed a gastric distention with fluid (Fig. 1A and B). Thoracic radiography showed no significant findings. Abdominal ultrasonography revealed a solitary, pedunculated, heterogeneous mass $(24.4 \times 24.5 \mathrm{~mm})$ 
arising from the mucosal layer and protruding within the lumen of the pylorus with intact wall layers. An evenly thick hypoechoic muscular layer (4 mm) was evident (Fig. 1C and D).

CT examination was performed using 32-multislice CT (Revolution; GE Healthcare, United Kingdom). The patient was positioned in ventral recumbency on the CT table under general anesthesia, having scanning parameters of $120 \mathrm{kV}, 85$ $\mathrm{mA}$, and $0.6 \mathrm{~mm}$ slice thickness. Contrast study was performed after intravenous administration of $600 \mathrm{mgI} / \mathrm{kg}$ iohexol (Bonorex 300 Inj.; Daehan Pharm, Korea) injected for 20 seconds using autoinjector (A-60; Nemoto Kyorindo Co., Japan), obtaining delayed phase postcontrast CT images 90 seconds after injection. A narrow-based, polypoid mass $(13.8 \times 23.3 \times 28.9$ $\mathrm{mm}$ ) in craniodorsal pyloric wall with remarkable homogeneous contrast enhancement and normal wall layers was detected (Fig. 2). A pyloric canal was narrowed (3 $\mathrm{mm}$ diameter on the sagittal plane). A splenic lymph node was round and enlarged $(6.7 \times 9.9 \mathrm{~mm})$ with normal contrast enhancement and a cyst-like component. Muscular layer hypertrophy in the pylorus and duodenum was identified. Based on radiography, ultrasound, and CT findings, differential diagnosis includes chronic hypertrophic pyloric gastropathy, gastric polyps, granulomatous gastritis, and less likely a gastric tumor.

After surgical excision of the mass, hypertrophic pyloric gastropathy with polypoid growth was histopathologically confirmed based on cystic hyperplasia of the gastric mucosal epi-
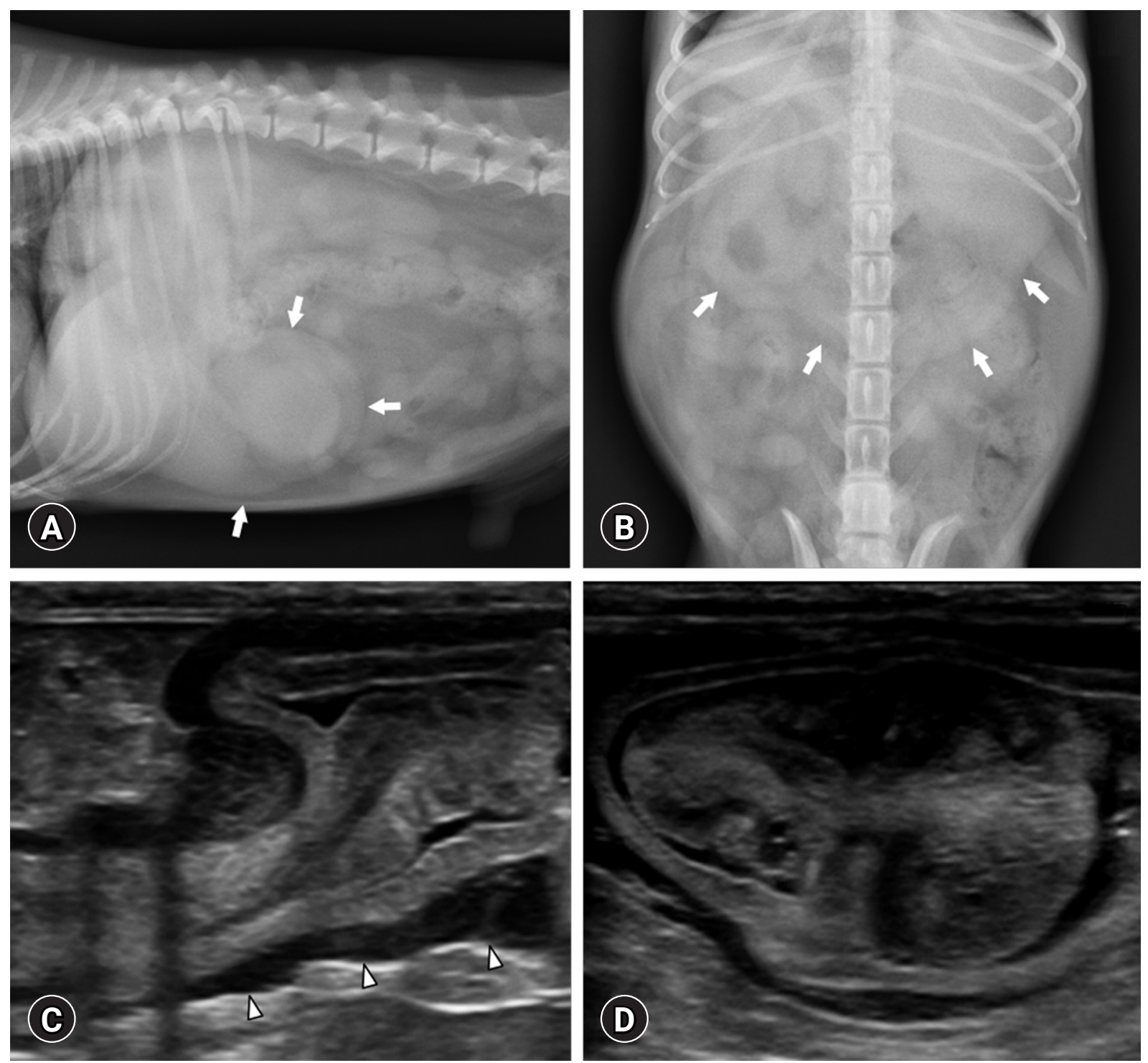

Fig. 1. Right lateral (A) and ventrodorsal (B) abdominal radiographs and ultrasonographic images of the stomach (C, D). Gastric distention (arrows) is identified. Solitary, pedunculated, heterogeneous mass arises from the mucosal layer and protrudes within the lumen in the pyloric lesion with intact wall layer (C, D). Pyloric muscle layer is evenly thickened (arrowheads). 

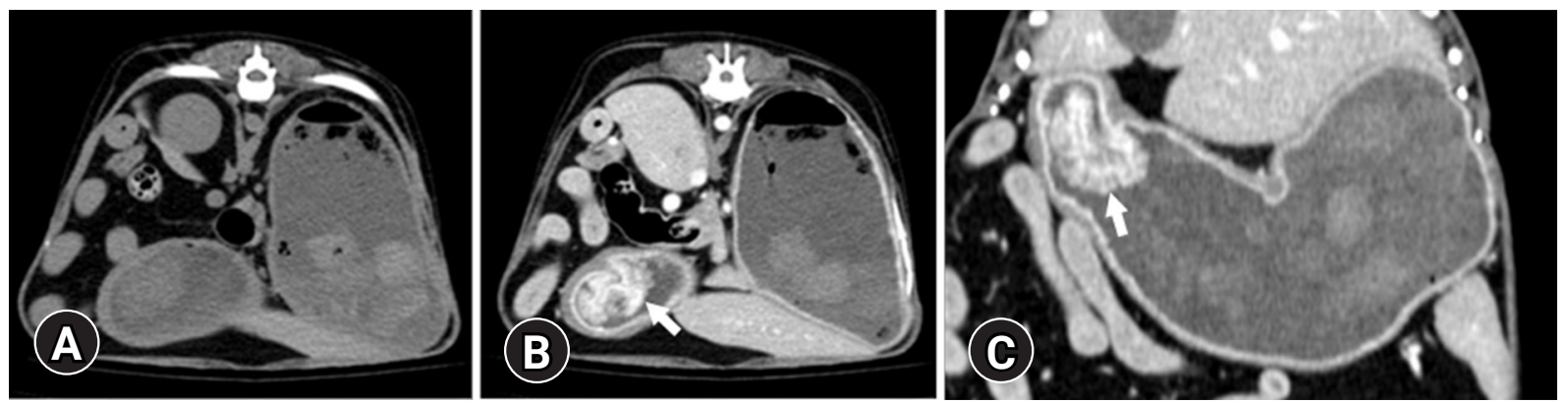

Fig. 2. Non-contrast (A) and postcontrast transverse (B), dorsal (C) CT images of the caudal abdomen. A narrow-based, polypoid mass (arrows) in craniodorsal pyloric wall is identified. The wall layer of the mass is intact and shows homogeneous contrast enhancement.
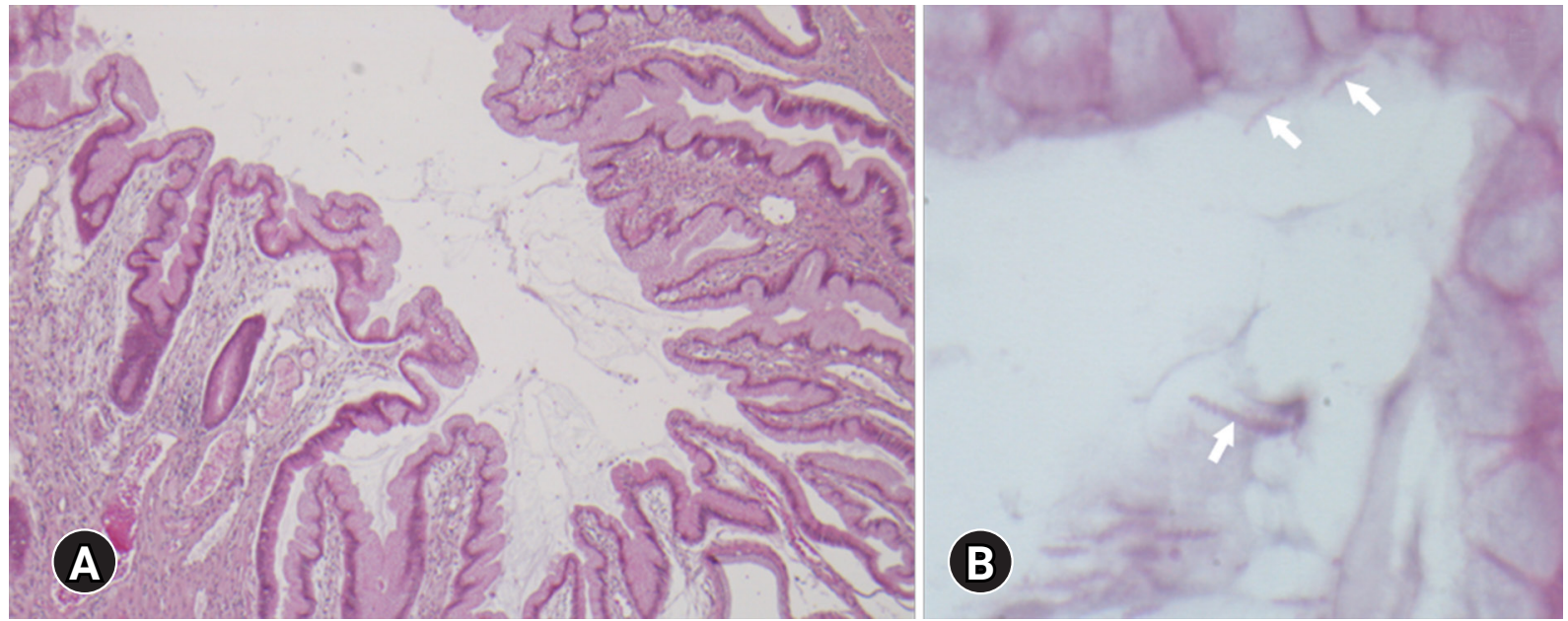

Fig. 3. Histopathological section of the pyloric mass. Cystic hyperplasia of the gastric mucosal epithelium, with thickened gastric muscularis and broad edematous lesions, and infiltration of chronic-active inflammatory cells are identified. Helical-shaped bacteria infiltrate the gastric mucosal epithelium (arrows) (hematoxylin-eosin stain; A: ×50, B: ×1,000).

thelium and infiltration of chronic-active inflammatory cells. Histological examination identified helical-shaped bacteria, Helicobacter spp., infiltrating the gastric mucosal epithelium (Fig. 3). After the 11-day follow-up, the dog was in good condition with no surgery complication.

Hypertrophic pyloric gastropathy causes the obstruction of the pyloric lumen or restriction of the luminal dilation and other conditions such as gastric polyps, granulomatous gastritis, tumors, pyloric foreign body, and intussusception [4]. These diseases' nonspecific clinical signs include vomiting, pain, and distention of the upper abdomen usually after a meal [5]. Nonobstructive hypertrophic pyloric gastropathy can be subclinical, when the pyloric canal is sufficiently patent, allowing gastric contents to empty into the duodenum despite being thickened [6]. Radiological findings are nonspecific for pyloric obstruction induced by hypertrophic pyloric gastropathy but can reveal a distended stomach with fluid or gas in the case of delayed gastric emptying $[1,5]$. The patient in the present study was considered to be nonobstructive hypertrophic pyloric gastropathy with nonspecific radiographic signs including mild gastric distension.

Ultrasound and CT are useful for differentiating the underlying disease for pyloric obstruction and grading the hypertrophic pyloric gastropathy $[1,5]$. In a previous study, hypertrophic pyloric gastropathy can be graded according to the distribution of gastric layers on histopathology and identified correlation histopathological findings with ultrasound [1]. Grade 1 is characterized by a thickened muscular layer, while grade 2 has both mucosal hyperplasia and muscular hypertrophy [1]. Grade 3 is characterized by mucosal hyperplasia with glandular and foveolar hyperplasia, glandular cystic dilatation, and mucosal/submucosal inflammation [1]. The classification system has a bearing on the appropriate surgical management of the condition [1]. In the same study, dogs with grades 1 or 2 show at least 
a 4-mm-thick muscular layer in the pyloric area on ultrasonography [1]. This study shows hypoechoic pyloric muscular layer thickness of $4 \mathrm{~mm}$ and confirms cystic hyperplasia of the gastric mucosal epithelium and chronic inflammatory cells through ultrasound and histopathology, respectively. The results show that the present case is a grade 3 hyperplastic pyloric gastropathy.

While the CT images of hypertrophic pyloric gastropathy of the previous study revealed a homogeneous contrast enhancement of the thickened gastric wall [5], the CT images of the present study showed a similar aspect of the contrast enhancement of the thickened gastric wall with intact gastric wall layering. However, our case showed mild enlarged splenic lymph node, whereas other previous studies revealed inflammation, such as giant hypertrophic gastritis with an enlarged gastric lymph node $[7,8]$. Similarly, the present study considered splenic reactive lymphadenopathy due to chronic inflammation.

Similar with hypertrophic pyloric gastropathy, larger neoplasia within the pylorus, such as adenocarcinomas and squamous cell carcinoma, can induce outflow obstruction $[4,9]$. The common ultrasound features of neoplasia include thickening of the stomach wall, distortion of the normal layering, and altered echogenicity and motility in the affected area [4]. Metastasis to the regional lymph node can be detected. Cytology or histopathology is required to differentiate inflammation from neoplastic condition and to categorize the tumor type [4]. On ultrasound and CT examination, intact gastric wall layer identification is important to differentiate hypertrophic pyloric gastropathy and gastric tumor $[4,5]$. On CT images, gastric tumor shows heterogeneous contrast enhancement, while hypertrophic pyloric gastropathy is homogeneously contrast enhanced like in the present study [5].

Histopathology in the present study confirms the presence of multiple helical-shaped bacteria infiltrating the gastric mucosal epithelium and chronic inflammatory cells, indicating Helicobacter spp. infiltration [10]. Helicobacter spp. have been identified as one of the most common causes of gastritis and peptic ulcer, although its etiology is not completely elucidated [10]. Helicobacter infection can stimulate increased release of gastrin and then induce acid secretion, causing gastric ulceration [11,12]. H. pylori infection induces hypertrophy of the gastric mucosa represented as a giant fold gastritis, by increasing apoptosis of surface and proliferative cells [13-15]; expands the proliferative cells; and affects the proliferative zone to the deeper gland as a compensatory response [13-15]. The correlation of Helicobacter spp. with the cellular proliferative activity and po- tential phenotypic alterations in canine spontaneous gastric polyps was investigated [13]. The presence of $H$. pylori seems to induce overexpression of cyclooxygenase- 2 in the deeper glands of gastric polyps, leading to an increased expression of this enzyme through the production of proinflammatory cytokines [13]. H. salomonis, H. felis, H. bizzozeronii, and H. heilmannii sensu stricto are the predominant gastric Helicobacter spp. in cats and dogs [14]. These non-H. pylori Helicobacter species have significant correlations with mild to moderate epithelial injury and mild to moderate intraepithelial lymphocyte infiltration of the canine stomach [14]. Based on previous studies, Helicobacter spp. and polypoid growth are related in this study.

In conclusion, an ultrasound of the gastric wall is useful for differentiating hypertrophic pyloric gastropathy from the other pyloric disease. The sonographic and CT features of hypertrophic pyloric gastropathy are intact wall layer and focal wall thickening. Helicobacter spp. in the stomach can induce hypertrophy or chronic inflammation. When thickened muscular layer with intact wall layering of the pylorus was identified on ultrasound and CT in dogs, chronic hypertrophic pyloric gastropathy should be considered in differential diagnosis. Helicobacter test (such as histopathology or kit) is suggested since the bacteria can cause the underlying inflammation. Long-term antibacterial therapy is needed for eradication.

\section{ORCID}

Hyunyoung Jang, https://orcid.org/0000-0002-8791-2517 Seoung-Woo Lee, https://orcid.org/0000-0002-7678-9242

Min Jang, https://orcid.org/0000-0002-2188-1906

Sungho Yun, https://orcid.org/0000-0002-9027-3859

Young-Sam Kwon, https://orcid.org/0000-0002-6489-0327

Jin-Kyu Park, https://orcid.org/0000-0003-4876-1055

Kija Lee, https://orcid.org/0000-0002-4649-809X

\section{References}

1. Biller DS, Partington BP, Miyabayashi T, Leveille R. Ultrasonographic appearance of chronic hypertrophic pyloric gastropathy in the dog. Vet Radiol Ultrasound 1994;35:30-33.

2. Bellenger CR, Maddison JE, MacPherson GC, Ilkiw JE. Chronic hypertrophic pyloric gastropathy in 14 dogs. Aust Vet J 1990;67:317-320.

3. Bayerdörffer E, Ritter MM, Hatz R, Brooks W, Ruckdeschel G, Stolte M. Healing of protein losing hypertrophic gastropathy by eradication of Helicobacter pylori--is Helicobacter pylori a 
pathogenic factor in Ménétrier's disease? Gut 1994;35:701704.

4. Stieger-Vanegas SM, Frank PM. The Stomach. In: Thrall DE (ed.). Textbook of Veterinary Diagnostic Radiology. 7th ed. pp. 894-925, Elsevier Health Science, St. Louis, 2018.

5. Rhim H, Moon S, Lee G, Park S, Cho KO, Choi J. Unusual ultrasonographic features of chronic hypertrophic pyloric gastropathy in two dogs. J Vet Clin 2018;35:302-307.

6. Stunden RJ, LeQuesne GW, Little KE. The improved ultrasound diagnosis of hypertrophic pyloric stenosis. Pediatr Radiol 1986;16:200-205.

7. Happé RP, van der Gaag I, Wolvekamp WT. Pyloric stenosis caused by hypertrophic gastritis in three dogs. J Small Anim Pract 1981;22:7-17.

8. Palumbo LT, Rugtiv GM, Cross KR. Giant hypertrophic gastritis: its surgical and pathologic significance. Ann Surg 1951; 134:259-267.

9. Seiler G, Mai W. The Stomach. In: O’Brien R, Barr F (eds.). BSAVA Manual of Canine and Feline Abdominal Imaging. pp. 87-109, British Small Animal Veterinary Association, Gloucester, 2009.

10. Leib MS, Duncan RB, Ward DL. Triple antimicrobial therapy and acid suppression in dogs with chronic vomiting and gas- tric Helicobacter spp. J Vet Intern Med 2007;21:1185-1192.

11. Lehmann FS, Schiller N, Cover T, Hatch R, Seensalu R, Kato K, Walsh JH, Soll AH. H.pylori stimulates gastrin release from canine antral cells in primary culture. Am J Physiol 1998;274: G992-G996.

12. Levi S, Beardshall K, Haddad G, Playford R, Ghosh P, Calam J. Campylobacter pylori and duodenal ulcers: the gastrin link. Lancet 1989;1:1167-1168.

13. Amorim I, Taulescu MA, Ferreira A, Rêma A, Reis CA, Faustino AM, Cătoi C, Gärtner F. An immunohistochemical study of canine spontaneous gastric polyps. Diagn Pathol 2014;9: 166.

14. Amorim I, Smet A, Alves O, Teixeira S, Saraiva AL, Taulescu M, Reis C, Haesebrouck F, Gärtner F. Presence and significance of Helicobacter spp. in the gastric mucosa of Portuguese dogs. Gut Pathog 2015;7:12.

15. Fukui T, Nishio A, Okazaki K, Uza N, Ueno S, Kido M, Inoue S, Kitamura H, Kiriya K, Ohashi S, Asada M, Tamaki H, Matsuura M, Kawasaki K, Suzuki K, Uchida K, Fukui H, Nakase H, Watanabe N, Chiba T. Gastric mucosal hyperplasia via upregulation of gastrin induced by persistent activation of gastric innate immunity in major histocompatibility complex class II deficient mice. Gut 2006;55:607-615. 